\title{
Microescultura da testa da semente madura de Ruellia elegans Poir. (Acan- thaceae)
}

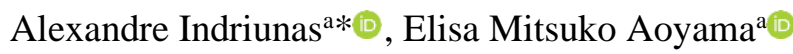 \\ a Universidade Federal do Espírito Santo, Brasil \\ *Autor correspondente (aleindri@gmail.com)
}

\section{N F O}

Keywords

helicoidal

thickenings Ruelliaea

trichomes

\section{Palavras-chaves}

espessamento

helicoidal

Ruelliaea

tricomas

\begin{abstract}
A B S T R A C T
Mature seed testa microsculpturing of Ruellia elegans Poir. (Acanthaceae).

Ruellia L. is the second largest genus of the Acanthaceae family and, like most of its representatives, has explosive loculicidal capsular fruits. In Acanthaceae, the morphology study, mainly of the microsculpture of seeds, aim to be important for taxonomic understanding and ecological relationships. R. elegans Poir. is an Atlantic Forest endemic species and used as ornamental. The present work aims to describe the morphology and seed testa microsculpture of $R$. elegans Poir. providing subsidies for the group's taxonomy and for ecological studies to understand seed dispersal patterns as well. The seeds were analyzed under scanning electron microscopy, and the description of the shape and indument was presented. The description and analysis of the mature seed testa microsculpture of the species corroborates its current taxonomic position due to the presence of mucilaginous trichomes throughout the surface and this characteristic indicates the ectozoochoric dispersion syndrome.
\end{abstract}

\section{R E S U M O}

Ruellia L. é o segundo maior gênero da família Acanthaceae e, como a maioria dos representantes dessa, possui frutos capsulares loculicidas explosivos. O estudo da morfologia e principalmente da microescultura das sementes em Acanthaceae tem se mostrado importante para compreensão das relações taxonômicas e ecológicas. R. elegans Poir. é uma espécie endêmica da Mata Atlântica emprega como ornamental. O presente trabalho tem por objetivo descrever a morfologia e microescultura da testa de sementes maduras de $R$. elegans Poir. fornecendo subsídios para a taxonomia do grupo, bem como para estudos ecológicos para o entendimento dos padrões de dispersão das sementes. As sementes foram analisadas sob microscopia eletrônica de varredura, sendo apresentada a descrição do formato e indumento. A descrição e análise da microescultura da testa da semente madura da espécie corroboram com seu atual posicionamento taxionômico devido à presença de tricomas mucilaginosos por toda a superfície e esta característica indica a síndrome de dispersão ectozoocórica. 


\section{INTRODUÇÃO}

Acanthaceae Juss. abrange 190 gêneros e mais de 4000 espécies predominantemente nas regiões tropicais (Mcdade et al., 2008), com 49 gêneros e 504 espécies para o Brasil (Profice et al., 2020). Ruellia L. é o segundo maior gênero da família Acanthaceae, com aproximadamente 350 espécies (Tripp et al., 2013) e para o Brasil são relatadas 84 (Profice et al., 2020).

Os frutos de Ruellia L., assim como da grande maioria das Acanthaceae, são cápsulas secas biloculadas, loculicidas e as sementes são fixadas em uma estrutura em forma de gancho (retinácula) a qual participa de ejeção daquelas quando da abertura do fruto (Ezcurra, 1993). O fruto capsular explosivo deiscente é uma importante sinapomorfia da família e a presença de retinácula é característica da maior subfamília Acanthoideae (Azevedo e Braz, 2018).

A morfologia juntamente com a microescultura da testa de sementes de Acanthaceae têm se provado relevantes em estudos de diversos táxons, em trabalhos como o percursor realizado de Schaffnit (1906) onde analisa 48 espécies de 22 gêneros, os de Balkwill e Campbell-Young (1999) com espécies de Thunbergia Retz. e Balkwill \& Schoonraad (1999) com Peristrophe Nees, além de regionais com Justicia L. da Tailândia (Ruengsawang et al.; 2012) e de Rhinacanthus Nees e Hypoestes Sol. ex R. Br. do Iêmen (Al-Hakimi et al., 2015). Quanto aos táxons neotropicais os trabalhos com Justicia de Peichoto (1998) e Aoyama et al. (2015), Herpetacanthus Nees (Indriunas et al., 2014) e do clado Mirandea (Justicieae, Tetramerium Lineage) (Kiel e Mcdade, 2014) endossam a importância destes estudos.

Ezcurra (1993) aponta a importância da morfologia das sementes na taxonomia de Ruellia L., o que é endossado por Greuter e Rodríguez (2010) nas Ruellinae de Cuba, Tripp e Fatimah (2012) no estudo de Satanocrater Schweinf, Tripp et al. (2013) em seu estudo sobre a tribo Ruellieae e Azevedo e Braz (2018) para as Ruellia de Mata Atlântica do Sudeste do Brasil.

Ruellia L. é o segundo gênero mais numeroso dentre as Acanthaceae com cerca de 350 espécies e o maior da tribo Ruellieae (Acanthoideae) (Tripp et al., 2013).

Dentre as espécies do gênero, Ruellia elegans Poir., endêmica do Brasil é bastante empregada como ornamental por suas flores vermelhas (Ezcurra, 1993; Profice et al., 2020). Tripp (2007) a posiciona no clado Ruellia inflata, o qual não possui sinapomorfias morfológicas conhecidas.

Ruellia elegans é um subarbusto ereto a decumbente, de 30-50 cm de altura, com folhas com 4-9 $\mathrm{cm}$ de largura por 2-5 $\mathrm{cm}$ de comprimento. Suas cápsulas são elíptico-obovadas com cerca de $2 \mathrm{~cm}$ de comprimento e podem conter mais de 12 sementes, as quais são achatadas, suborbiculares, com 2$3 \mathrm{~mm}$ de largura, adpresso-pubescentes (Ezcurra, 1993).

Segundo Profice et al. (2020) a espécie ocorre na Mata Atlântica em Floresta Estacional Semidecidual e Floresta Ombrófila, dos estados de Goiás, Minas Gerais, São Paulo e Paraná. Ezcurra (1993), por sua vez, assinala que ela é encontrada em áreas perturbadas, mais ou menos abertas, porém devido ao seu uso ornamental é amplamente cultivada.

O presente trabalho tem por objetivo descrever a morfologia e microescultura da testa de sementes maduras de Ruellia elegans Poir. fornecendo subsídios para a taxonomia do grupo, bem como para estudos ecológicos para o entendimento dos padrões de dispersão das sementes.

\section{MATERIAL E MÉTODOS}

As sementes maduras de R. elegans foram coletadas de 10 indivíduos cultivados propagados espontaneamente em jardim residencial no município de Taubaté, estado de São Paulo, Brasil (-45.5483 $23^{\circ} 1^{\prime} 51^{\prime \prime} \mathrm{S}, 45^{\circ} 32^{\prime} 54^{\prime \prime} \mathrm{W}$ ), as plantas foram cultivadas conforme indicado por Lorenzi e Souza (2008). O voucher foi depositado no Herbário SAMES (n 8642).

Para a descrição da microescultura da testa, as sementes maduras após desidratação em série etílica, foram secas ao ponto crítico com $\mathrm{CO}_{2}$, fixadas em suporte de alumínio com fita dupla face de carbono e metalizadas com uma camada de ouro de $5 \mathrm{~nm}$ a $1,5 \mathrm{~mm}$ no equipamento modelo Quorum Q 150TE. As análises e a digitalização das imagens foram realizadas ao microscópio eletrônico de varredura modelo Zeiss - EVO LS15. Esta etapa foi realizada no Laboratório de Microscopia Eletrônica de Varredura do Departamento de Física e Química (DFQ) da Universidade Estadual Paulista "Julio Mesquita Filho" (UNESP) campus de Ilha Solteira, São Paulo. A terminologia empregada para a descrição da microescultura da testa da semente foi baseada em Payne (1978).

\section{RESULTADOS E DISCUSSÃO}

As sementes são achatadas dorso ventralmente, elípticas a obovadas, levemente comprimidas na borda (Figs 1A, 1B, 1E, 1F, 1G), ápice arredondado (Fig 1A) a acuminado (Figs 1B, 1C, 1D), com a base assimétrica na área da micrópila com um estreitamento na porção final da calaza (Fig $1 \mathrm{~A})$. Estes aspectos corroboram com o apresentado por Ezcurra (1993) e segundo Greuter; Rodríguez 
(2010) o formato varia conforme a posição no fruto, não possuindo assim valor taxonômico.

Como pode ser observado (Fig 1) há a presença conspícua de tricomas mucilaginosos em toda a superfície da semente, cobrindo-a completamente, incluindo o ápice (Fig 1D) e toda a margem (Fig 1F, $1 \mathrm{G})$. A presença destas estruturas em toda a superfície corrobora com o assinalado por Tripp et al. (2013) onde a distribuição destes tricomas possuem valor taxonômico para a tribo Ruellieae, endossando o posicionamento da espécie no clado " $R u$ ellia inflata" sensu Tripp e McDade (2013), que segundo as autoras não possui sinapomorfias morfológicas conhecidas (Azevedo e Braz, 2018).

Os tricomas mucilaginosos possuem espessamento helicoidal de parede (Figs 1F, 1H, 1I, 1J), onde pode se observar o aspecto de véu devido à presença da mucilagem na parede (Fig 1J). Este formato já foi descrito em diversos trabalhos (Schaffnit, 1906; Greuter e Rodríguez, 2010; Tripp e Fatimah, 2012). Muito embora a espécie em estudo já tenha sido tratada em trabalho anterior, como $R$. fragans Andr., (Schaffnit, 1906) a avaliação em microscopia eletrônica de varredura fornece maior detalhamento. Cabe salientar que as formas bem como características morfológicas da testa dependem da análise de material plenamente desenvolvido (Ruengsawang et al., 2012; Indriunas et al., 2014; Aoyama et al., 2015).

Uma das mais notáveis características das sementes de Ruelliaea é a presença de tricomas mucilaginosos, como observado em detalhes na Figura 1J. A mucilagem secretada pelos tricomas possui importante papel na dispersão ectozoocórica pela forte adesão que propicia (Ezcurra, 1993; Greuter e Rodríguez, 2010) e pela sua capacidade higroscópica parece atuar como fator relevante na germinação (Greuter e Rodríguez, 2010). Trabalhos com a caracterização destas estruturas tem se mostrado relevantes para o entendimento de sua função (Schnept e Deichgräber, 1983; Greuter e Rodríguez, 2010).
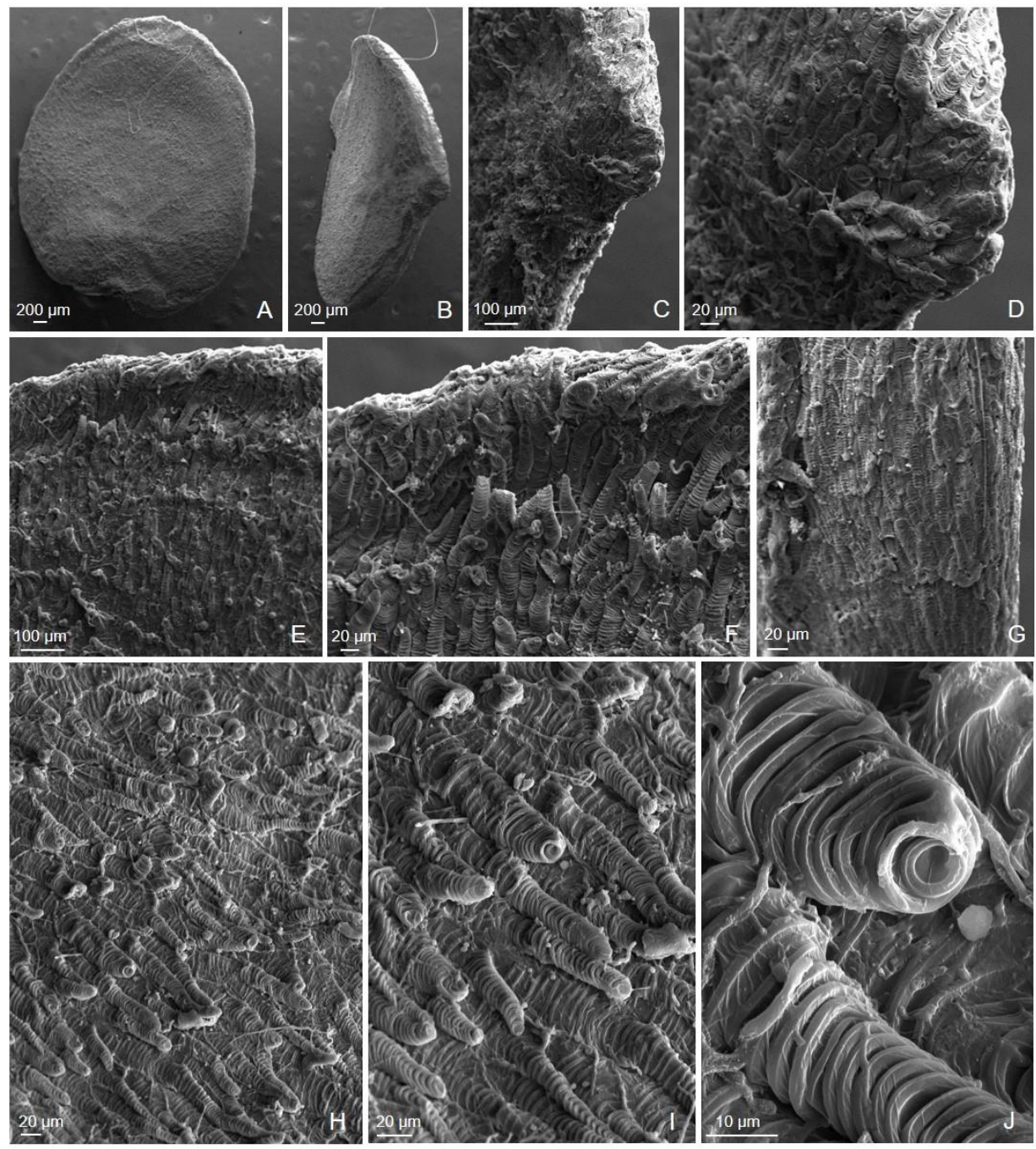

Figura 01. A. Aspecto geral, vista frontal. B. Aspecto geral, vista superior da região apical. C-D. Detalhe do ápice. E -G. Detalhe da margem. H- J. Detalhe da superfície evidenciando tricomas mucilaginosos. 


\section{CONCLUSÕES}

A descrição e análise da microescultura da testa da semente madura de $R$. elegans, por microscopia eletrônica de varredura, vem a contribuir para o conhecimento desta estrutura para a família, mais especificamente para Ruellieae nativas. A presença de tricomas mucilaginosos por toda a superfície confirma seu posicionamento nos estudos taxonômicos atuais (clado "Ruellia inflata") e estas estruturas indicam a síndrome de dispersão por ectozoocoria. Deste modo o presente trabalho contribui para o maior conhecimento da espécie estudada.

\section{REFERÊNCIAS BIBLIOGRÁFICAS}

Al-Hakimi AS, Maideen H., Latiff A. Pollen and Seed Morphology of Rhinacanthus Nees and Hypoestes Sol. ex R. Br. (Acanthaceae) of Yemen. Sains Malaysiana, v. 44, n. 1, p. 715, 2015.

Aoyama EM, Indriunas A, Vitória EL, Monteiro MM. Caracterização morfológica de frutos e sementes de Justicia scheidweileri. Natureza on line, v. 13, p.3, p. 134-140, 2015.

Azevedo IHF, Braz DM. Seed morphology of Ruellia L. (Acanthaceae) from the Southeastern Brazilian Atlantic rain forest: Taxonomic, phylogenetic, and ecological. Flora, v. 240, p. 48-57, 2018. https://doi.org/10.1016/j.flora.2017.12.011

Balkwill K, Campbell-Young G. Taxonomic studies in Acanthaceae: testa microsculpturing in southern African species of Thunbergia. Botanical Journal of Linnean Society, v. 131, p. 301-325, 1999.

Balkwill K, Schoonraad E. Taxonomic studies in Acanthaceae: testa microsculpturing in southern African species of Peristrophe. South African Journal of Botany, v. 52, p. 513520, 1999.

Ezcurra C. Systematics of Ruellia (Acanthaceae) in southern South America. Annals of the Missouri Botanical Garden, v. 80, p. 787-845, 1993.

Greuter W, Rodríguez RR. Notes on some endemic Cuban species of Ruelliinae (Acanthaceae), on their seeds, pollen morphology and hygroscopic features. Willdenowia, v. 40, P. 285-304, 2010. https://doi.org/10.3372/wi.40.40210

Indriunas A, Aoyama EM, Kameyama C. Microescultura da testa da semente madura de Herpetacanthus chalarostachyus Indriunas \& Kameyama (Acanthaceae). Natureza on line, v. 12, n. 1, p. 48-50, 2014.

Kiel CA, MCDADE LA. The Mirandea Clade (Acanthaceae, Justicieae, Tetramerium Lineage): phylogenetic signal from molecular data and micromorphology Makes Sense of taxonomic confusion caused by remarkable diversity of floral form. Systematic Botany, v. 39, p. 950-964, 2014. https://doi.org/10.1600/036364414X681446

Lorenzi H, Souza HM. Plantas ornamentais no Brasil: arbustivas, herbáceas e trepadeiras. São Paulo: Nova Odessa. 2008.

Payne WW. A glossary of plant hair terminology. Brittonia, v. 30, n. 2, p. 239-255, 1978.

Peichoto MC. Estudios morfo-anatómicos em espécies de Justicia (Acanthaceae) del nordeste argentino. Darwiniana, v.
36, p. 13-28, 1998

Ruengsawang K, Chantaranothai P, Simpson DA. Contributions to the seed morphology and taxonomy of Justicia (Acanthaceae) from Thailand. Journal of Systematics and Evolution, v. 50, p. $153-162, \quad 2012$. https://doi.org/10.1111/j.1759-6831.2012.00178.x

Schaffnit E. Beiträge zur Anatomie der Acanthaceen-Samen. Beihefte zum Botanischen Centralblatt, Abt. 1, v. 19, p. 453 $-521,1906$.

Schnepf E, Deichgräber G. Structure and formation of fibrillar mucilages in seed epidermis cells: II. Ruellia (Acanthaceae). Protoplasma, v. 114, p. 222-234, 1983.

Tripp EA. Evolutionary relationships within the species-rich genus Ruellia (Acanthaceae). Systematic Botany, v. 32, p. 628-649,

2007. https://doi.org/10.1600/036364407782250625

Tripp EA, Daniel FD, Fatimah S, Mcdade LA. Phylogenetic relationships within Ruellieae (Acanthaceae) and a revised classification. International Journal of Plant Sciences, v. 174, p. 97-137, 2013. https://doi.org/10.1086/668248

Tripp EA, Fatimah S. Comparative anatomy, morphology, and molecular phylogenetics of the african genus Satanocrater (Acanthaceae). American Journal of Botany, v. 99, n. 6, p. 967-982, 2012. https://doi.org/10.3732/ajb.1100354

Tripp EA, Mcdade LA. Time-calibrated phylogenies of hummingbirds and hummingbird-pollinated plants reject a hypothesis of diffuse co-evolution. Aliso, v. 31, n. 2, p. 89103, 2013. 